\title{
La pirotecnia y todas sus consecuencias. Síndrome de Blast: reporte de un caso
}

\section{The pyrotechnics and all the consequences. Blast Syndrome. Case Report}

\author{
Manuel Gil-Vargas, ${ }^{1}$ Yaneth Martínez-Tovilla, ${ }^{2}$ Magdalena León-López, ${ }^{3}$ Mariana Lee Miguel-Sardaneta, ${ }^{4}$ César \\ Llanos-Herrea, ${ }^{5}$ Estefanía Martín-Zayago, ${ }^{5}$ Johana Ramírez-Cruz ${ }^{6}$
}

\section{Resumen}

ANTECEDENTES: El síndrome de Blast es provocado por la exposición del organismo a los efectos de una onda de choque ocasionada por una explosión. En México, los juegos pirotécnicos representan un riesgo de sufrir una lesión traumática.

CASO CLínICO: Adolescente de 14 años, con múltiples complicaciones derivadas de la explosión de una "paloma". Requirió cirugía exploratoria abdominal indicada por datos de abdomen agudo posterior a la explosión.

CONCLUSIONES: El síndrome de Blast es un padecimiento poco identificado e infradiagnosticado que causa lesiones fatales y múltiples consecuencias que, en la mayoría de los casos, pueden prevenirse.

PALABRAS CLAVE: Explosión; pirotecnia; politraumatismo; complicaciones; prevención.

Abstract

BACKGROUND: Blast syndrome is a clinical syndrome caused by exposure of the organism to the effects of a shock wave caused by an explosion. The pyrotechnic games in Mexico directly influence the risk of suffering a traumatic injury, as happens with the use of explosive objects prepared with gunpowder.

CLINICAL CASE: We report the case of 14 -year-old adolescent who suffered multiple complications because of the explosion of one of these objects called "dove", required abdominal exploratory surgery for presenting acute abdomen data after the explosion. CONCLUSION: Blast syndrome is a poorly identified and underdiagnosed condition that causes fatal injuries and multiple consequences, which in most cases is preventable. KEYWORDS: Explosion; Pyrotechnics; Polytrauma; Complications; Prevention.
${ }^{1}$ Servicio de Cirugía Pediátrica, Hospital General de Puebla Eduardo Vázquez Navarro.

2 Pediatra intensivista, Unidad de Quemados de los Servicios de Salud del Estado de Puebla, profesor investigador de tiempo completo, Facultad de Medicina, Benemérita Universidad Autónoma de Puebla.

${ }^{3}$ Pediatra intensivista, Hospital General de Puebla Eduardo Vázquez Navarro.

${ }^{4}$ Pediatra, Hospital General de Puebla, Zona Norte.

${ }^{5}$ Médico pasante en Servicio Social Facultad de Medicina, Benemérita Universidad Autónoma de Puebla.

${ }^{6}$ Residente de Cirugía pediátrica, Hospital para El Niño Poblano, Puebla, Pue.

Recibido: 12 de julio 2018

Aceptado: 21 de septiembre 2018

Correspondencia

Manuel Gil Vargas

gilvm@yahoo.com

Este artículo debe citarse como Gil-Vargas M, Martínez-Tovilla Y, LeónLópez $M$, Lee Miguel-Sardaneta $M$ Llanos-Herrea C, Martín-Zayago E, Ramírez-Cruz J. La pirotecnia y todas sus consecuencias. Síndrome de Blast: reporte de un caso. Acta Pediatr Mex. 2019;40(1):16-21.

\section{ANTECEDENTES}

El síndrome de Blast es el conjunto de lesiones producidas como consecuencia de la propagación de una onda explosiva generada por un foco explosivo a través del organismo. La explosión crea una rápida expansión de gas, genera aumento de la presión y una onda de viento que se desplaza, y la formación de una esfera de alta temperatura. ${ }^{1,2}$ Las lesiones provocadas se dividen en: primarias: por cambios de presión que afectan a los órganos con contenido de aire (oído, pulmón, vísceras). Secundarias: por proyectiles desplazados de la explosión (traumatismo penetrante). Terciarias: el individuo es desplazado por los vientos de la explosión y arrojado contra 
objetos fijos. Cuaternarias: causa directa de la explosión, exacerbaciones o complicaciones (quemaduras, inhalación de gases y asfixia).1-4 En México se dispone de escasa información acerca de este síndrome, que es decisivo porque afecta a numerosos órganos del cuerpo, provoca amputaciones traumáticas, discretas microperforaciones ${ }^{2}$ que requieren una exploración física minuciosa y búsqueda intencionada; su desenlace puede ser fatal si no se tratan oportunamente. Los accidentes en niños son una de las principales causas de morbilidad y mortalidad en menores de 15 años, representan un problema de salud pública con un costo social y económico importante derivado de las lesiones originadas.

\section{CASO CLÍNICO}

Adolescente masculino, de 14 años, sin antecedentes de importancia para el caso. El padecimiento se inició el primero de abril de 2018, en su domicilio, al manipular con un desarmador un artefacto pirotécnico, coloquialmente conocido como "paloma", sin ser encendido. Debido a la fricción explotó y le provocó una amputación traumática de la mano izquierda y quemaduras de segundo grado superficial en el hipogastrio, área púbica y genital (Figura 1). Lo atendieron en el Hospital Regional de Tecomatlán, Puebla, donde le operaron el muñón de la extremidad afectada y referido 24 horas después a la unidad de quemados de los Servicios de Salud del Estado de Puebla.

\section{Cronología de eventos}

Se recibió en el área de urgencias consciente, cooperador y ojos con hiperemia conjuntival. Abdomen blando, no doloroso, depresible, peristalsis, quemaduras en el pubis y área genital. Se le realizaron: impresión inicial, evaluación primaria y secundaria, curación de las quemaduras y ultrasonido (Focused Abdominal Sonography for Trauma-Extended (FAST-E), sin reporte de alteraciones ni líquido libre en la cavidad abdominal

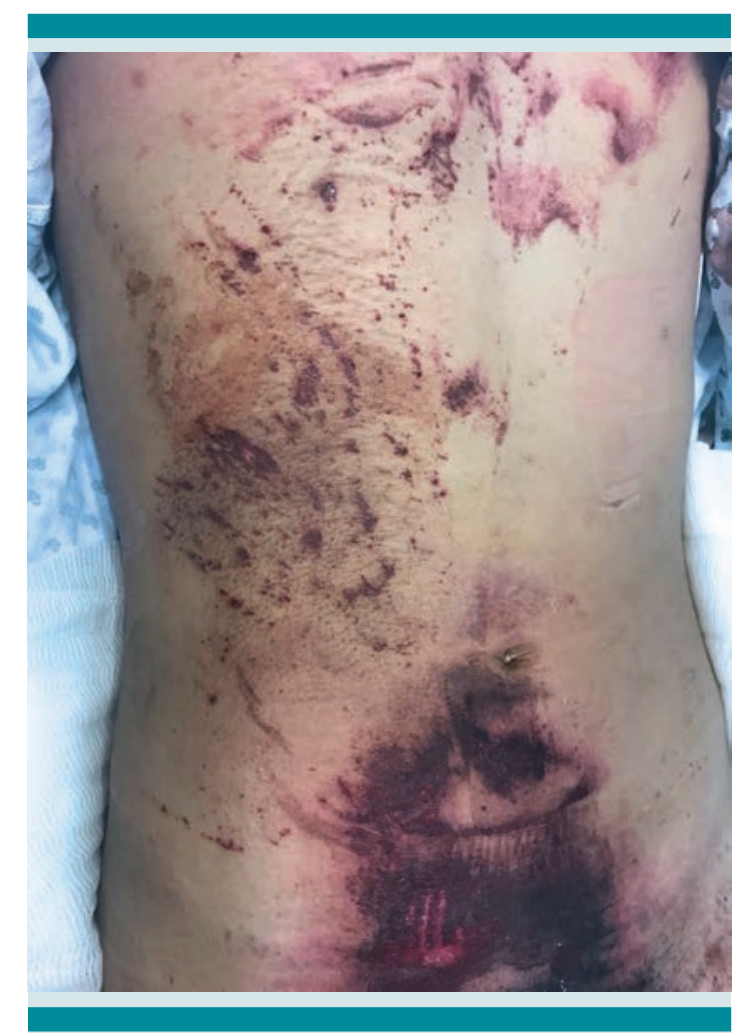

Figura 1. Abdomen con quemaduras de segundo grado, superficiales, con bordes irregulares, en el hipogastrio y región genital.

al momento de su ingreso. Se hospitalizó para observación; posteriormente se intervino para aseo quirúrgico. Se le administraron ceftriaxona y clindamicina indicadas por las condiciones de la lesión y el entorno en el que se produjeron. Aproximadamente seis horas después del ingreso inició con dolor abdominal periumbilical y distensión abdominal, con peristalsis.

Ante la imposibilidad de la bipedestación, los médicos de la unidad de quemados solicitaron una radiografía abdominal, con rayo tangencial, en la que se observó una adecuada distribución de gases, sin niveles hidroaéreos. Doce horas después el paciente refirió incremento del dolor abdominal, irradiado a ambos flancos, distensión abdominal y ausencia de peristalsis. Se repitió el ultrasonido FAST-E y se observó un área hipe- 
recoica entre la cara inferior del lóbulo derecho hepático y el polo superior del riñón derecho (Figura 2). Se tomó nueva radiografía de abdomen que mostró los pliegues intestinales engrosados, lisos, rectos y perpendiculares al eje longitudinal, y una imagen radiolúcida, compatible con aire libre en la cavidad abdominal. Posteriormente se realizó una tomografía axial computada abdominal, contrastada, en la que se observó la pared abdominal sin engrosamiento, con líquido isodenso desde el espacio de Morrison y corredera parietocólica derecha, acumulado en la pelvis. Figura 3

Un cirujano pediatra efectuó una exploración quirúrgica abdominal y encontró isquemia intestinal a $70 \mathrm{~cm}$ del ángulo de Treitz y seis perforaciones puntiformes con salida de líquido biliar, en un segmento de $30 \mathrm{~cm}$. Se practicaron la anastomosis término-terminal y el lavado exhaustivo de la cavidad abdominal. Por cambios isquémicos en trayecto proximal y distal a la anastomosis, e inestabilidad hemodinámica, se decidió el manejo de abdomen abierto con bolsa de Bogotá, durante 72 horas, y permanencia en el servicio de terapia intensiva del Hospital para el Niño Poblano.

\section{Seguimiento y resultados}

Se llevó a cabo una nueva exploración quirúrgica 72 horas después de la primera y se revisó desde el ángulo de Treitz hasta la válvula ileocecal y anastomosis íntegra, sin evidencia

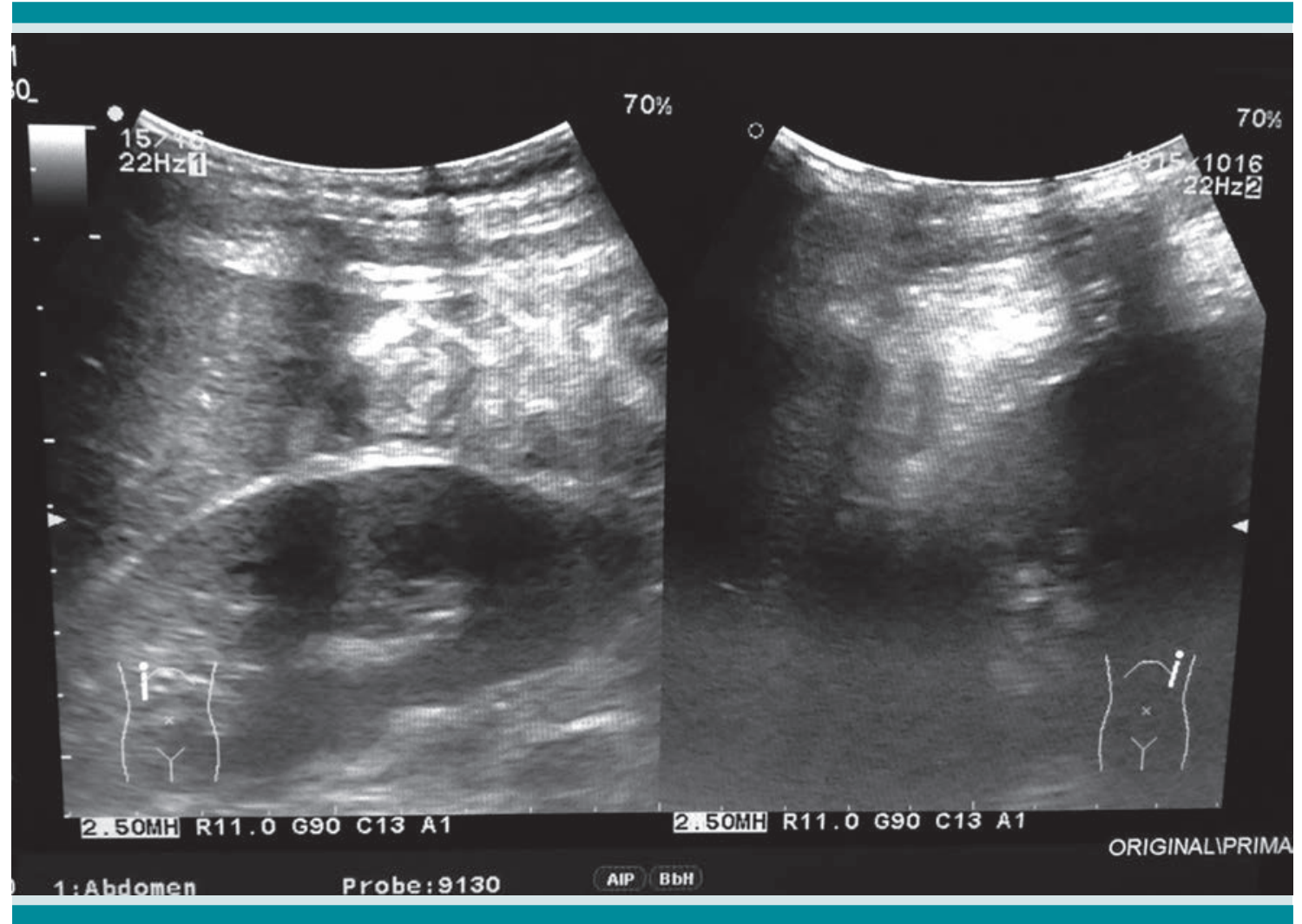

Figura 2. USG FAST-e, se observa un área hiperecoica entre la cara inferior del lóbulo derecho hepático y el polo superior del riñón derecho; es la imagen clásica de líquido libre en el espacio de Morrison. 


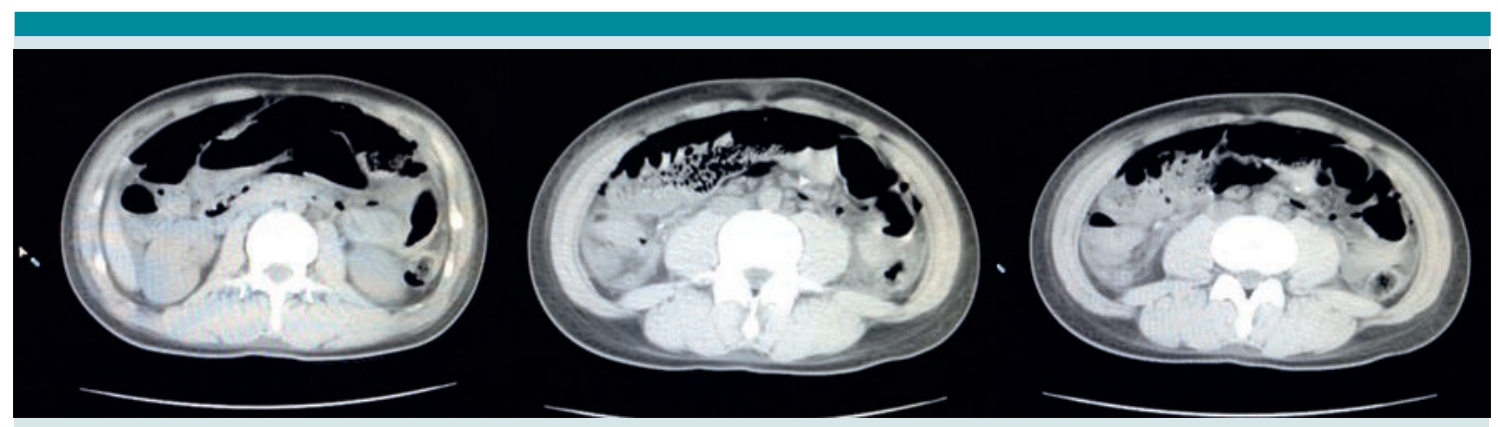

Figura 3. Pared abdominal sin engrosamiento; líquido isodenso desde el espacio de Morrison, corredera parietocólica derecha, acumulación en la pelvis.

de perforación intestinal. Reporte de patología: perforación intestinal y peritonitis aguda secundaria. Durante la estancia hospitalaria de cuatro días permaneció con sedo-relajación, después con inversión del ciclo vigila-sueño, mutismos y agitación psicomotriz, tratado por paidopsiquiatría con risperidona, con diagnóstico de estrés postraumático. Recibió apoyo aminérgico, con adrenalina y norepinefrina, durante 4 días, con estado de choque séptico con foco abdominal, enseguida se destetó de las aminas. Permaneció en fase III de la ventilación durante 4 días, posteriormente con oxígeno suplementario por espacio de 48 horas y destete de éste sin eventualidades. Recibió alimentación enteral vía sonda nasogástrica, con evacuaciones a las 48 horas de la alimentación enteral, con pérdida de peso de 8 kg durante su estancia; mostró recuperación nutricional con adición de dieta polimérica. Se trató con clindamicina y metronidazol durante 21 días, más fluconazol por espacio de 14 días debido a la existencia de levaduras en el líquido peritoneal. Tuvo infección de la herida quirúrgica en plano superficial, que se controló con curación diaria de la herida y esquema de antibiótico.

\section{DISCUSIÓN}

El paciente fue víctima de una explosión fortuita de un cohete, que en México se venden sin control alguno, incluso a menores de edad. Estos explosivos se distribuyen en establecimientos improvisados o de manera ambulatoria, se fabrican artesanalmente, sin control de calidad. La pirotecnia juega un papel decisivo en la idiosincrasia mexicana y es parte de distintas celebraciones religiosas e históricas. ${ }^{5}$

Los explosivos están regulados por la Ley Federal de Armas de Fuego y Explosivos, en la que se especifica que las actividades industriales y comerciales relacionadas con éstos, se sujetarán a las disposiciones que dicte la Secretaría de la Defensa Nacional, requiriendo permisos otorgados por la misma para realizar actividades relacionadas con pirotecnia. Los fuegos artificiales obtenidos en lugares autorizados se consideran de muy bajo riesgo y de libre venta al público en general (hasta $10 \mathrm{~kg}$ ), sin necesidad de contar con algún permiso para su obtención. En México se decomisan y clausuran, permanentemente, bodegas que almacenan pólvora blanca, que es sumamente inestable y estalla con el mínimo roce, golpe o aumento de temperatura. Su onda expansiva es capaz de provocar las lesiones comentadas.

Por desgracia, no obstante estar penado con hasta 15 años de prisión a todo aquél que fabrique o exporte armas, municiones, cartuchos o explosivos sin el permiso correspondiente, cada año se clausuran y decomisan una gran cantidad de 
explosivos clandestinos. Estos llegan a manos de una variada población, ${ }^{6}$ incluso a la pediátrica, que en muchas ocasiones ya padece enfermedades que complicarán el enfoque terapéutico. Si a lo anterior se agrega la falta de equipo de protección, la posibilidad de traumatismos severos en todos los sistemas se incrementa de manera exponencial. ${ }^{7}$

La fuerza explosiva se trasmite a través de la pared del aparato gastrointestinal hacia las distintas vísceras, moviliza el aire intestinal a una presión muy elevada y ocasiona fenómenos mecánicos que afectan a las vísceras huecas. Pueden sobrevenir hemorragias gástricas o intestinales, subserosas o submucoas, estallidos y perforaciones de las vísceras abdominales huecas, estallidos y desgarros de vísceras macizas. La perforación intestinal suele ser tardía y agregarse hipoperfusión mesentérica por las lesiones de cizallamiento o de embolia gaseosa que provocan necrosis isquémica del intestino. ${ }^{8}$

El síndrome de Blast origina múltiples lesiones que pueden repercutir a largo plazo, no solo orgánicamente, sino en ámbitos psicológicos y psiquiátricos, pues este padecimiento es un detonante de estrés postraumático ${ }^{2,9,10,11}$ y económico debido a la variedad de lesiones, y a la asociación con una estancia hospitalaria prolongada y reingresos del paciente. Tiene también repercusiones sociales porque los niños afectados suelen tener dificultad para reincorporarse a la vida cotidiana de manera normal debido a las cicatrices o amputaciones. Es importante crear conciencia en los padres, cuidadores, autoridades, los propios niños y la sociedad civil en general a fin de prevenir este tipo de accidentes. Regular y supervisar la venta de explosivos, y fomentar que solamente pueden manipularlos y encenderlos personas calificadas, para evitar detonaciones accidentales y las complicaciones que puedan originar. ${ }^{6}$

\section{CONCLUSIONES}

El síndrome de Blast suele pasar inadvertido y es subestimado debido a una baja sospecha. Al tratar a un paciente herido por pirotecnia, las lesiones visibles (quemaduras) pierden importancia en comparación con las causadas por los cuatro mecanismos del síndrome de Blast. Las lesiones accidentales constituyen una de las principales causas de amenaza para la vida de la población infantil. Por esto, la prevención debe considerarse una prioridad de salud y social, tanto por la elevada mortalidad, morbilidad y discapacidad que originan, como por el elevado costo económico que representa su atención.

Los accidentes son sucesos imprevistos que ocasionan lesiones y muerte, en la mayoría de los casos susceptibles de prevención. Ésta siempre será más efectiva si se fomenta en edades tempranas, concientizando a los menores de los peligros que pueden correrse si los explosivos se manipulan sin el debido adiestramiento y precausiones. Lo más importante es crear la conciencia que el alejamiento de los mismos es la mejor prevención que evitará los accidentes.

\section{REFERENCIAS}

1. Westrol M, Donovan C, Kapitanyan R. Blast Physics and Pathophysiology of Explosive Injuries. Ann Emerg Med. 2017; 69:S4-S9.

2. Pasquier $P$, Lenoir $B$, Debien B. Blast y lesiones por explosión. EMC Anestesia-Reanimación 2014; 40(1):1-12.

3. Giménez de Azcárate $C$, Baigorri Soler M. Muerte por explosión: cuestiones y sistemática médico-forenses. Cuadernos de Medicina Forense 2002; 27 :39-52.

4. Zurbarán-Barrios M, Calle J, Restrepo R. Descripción de lesiones por explosivos: revisión de la literatura, reporte de caso y propuesta de nueva clasificación. Rev. Case Rep. 2015;1 (Suppl. 1):168-84.

5. Dávila E, Martínez R, Martínez E, Hernández E, Vargas R. Pirotecnia Mexiquense Artesanía de fuego. 2a ed. Toluca: Consejo Editorial de la Administración Pública Estatal, 2013.

6. Ley Federal de Armas de Fuego y Explosivos. Última reforma DOF 12-11-2015: 1-32. 
Gil-Vargas M, et al. Síndrome de Blast

7. Díaz A, Balparda K. Lesión ocular secundaria a trauma por explosión: lo que el médico de urgencias debe saber. Archivos de Medicina de Urgencia de México. 2013; 5: 70-77.

8. Pasquier P, Lenoir B, Debien B. Blast y lesiones por explosión. EMC - Anestesia-Reanimación. 2014; 40:1-11.

9. Tschiffely A, Ahlers S, Norris J. Examining the relationship between Blast-induced mild traumatic brain injury and posttraumatic stress-related traits. Journal of Neuroscience Research. 2015;93:1769-77.

10. Rosenfeld J, McFarlane A, Bragge P, et al. Blast-related traumatic brain injury. Lancet Neurol. 2013; 12: 882-93.

11. Reid M, Velez C. Discriminating military and civilian traumatic brain injuries. Molecular and Cellular Neuroscience. 2015;66:123-28. 